\title{
Treatment of obstructive sleep-disordered breathing with positive airway pressure
} systems

\author{
D. Pevernagie*, J.F. Masa”, J.C. Meurice ${ }^{\ddagger}$, R. Farré$^{+}$, O. Marrone ${ }^{\S}$ and D. Rodenstein ${ }^{\dagger}$
}

ABSTRACT: Positive airway pressure systems are widely used to treat patients with moderate-tosevere obstructive sleep-disordered breathing. The application of stable continuous positive airway pressure (CPAP) via the nose (nasal CPAP) has been the mainstay of treatment since the early 1980s. For treatment to be effective, the pressure level must be fine-tuned to restore patency of the individual patient's upper airway.

Currently, there is ongoing controversy concerning which outcomes to observe when adapting the pressure level, and which methods to use for pressure adaptation. Adjusting the pressure level to control apnoeas and hypopnoeas is one major objective, but may not be sufficient to restore normal sleep. Evidence is available that elimination of inspiratory flow limitation leads to better results.

In recent years, it has become evident that the use of empirically set CPAP or automatic CPAP devices parallel the clinical results obtained with the classical approach of manual CPAP titration. A striking and still unexplained paradox lies in the fact that automatic CPAP devices perform very differently on the bench, but still yield satisfactory results on several clinical outcomes, e.g. control of sleep-related respiratory disturbances, restoration of good sleep quality and daytime alertness. Understanding the functioning of automatic CPAP devices can prove difficult, as the mode of operation is usually not disclosed by the manufacturers.

At present, it is impossible to make any scientifically sound statement on the appropriateness of using automatic continuous positive airway pressure devices for the routine treatment of patients with obstructive sleep-disordered breathing. For this purpose, convincing results of phase I-III clinical trials are needed.

KEYWORDS: Automatic continuous positive airway pressure, continuous positive airway pressure, sleep apnoea, sleep-disordered breathing

$\mathbf{N}$ asal continuous positive airway pressure (CPAP) has been the mainstay for treatment of patients suffering from the obstructive sleep apnoea/hypopnoea syndrome (OSAHS), ever since its introduction in 1981 by Sullivan et al. [1]. Nasal CPAP acts as a pneumatic splint that prevents passive collapse of the upper airway. To be effective, the level of nasal CPAP must exceed the critical closing pressure of the upper airway [2]. This concept is crucial to the contention that titration of nasal CPAP should be carried out to determine the exact "dose" of pressure that will prevent upper airway obstruction in all sleep states and body postures [3]. Thus, data from CPAP titration studies are required for the prescription of fixed CPAP (F-CPAP) at therapeutically effective levels. Based on this common belief, polysomnographic studies for pressure assessment have been performed in many sleep laboratories all over the world, from the early 1980s onwards. While scientific proof for the need of CPAP titration studies was never provided, it has become general practice. Two other approaches for initiating CPAP treatment have been introduced in recent years. Prediction formulae based on simple anthropometric and sleep variables have been developed for the estimation of therapeutic pressure levels $[4,5]$. In a study by OLIVER and HOFFSTEIN [6], predicted values were shown to approximate titrated CPAP levels within a range of $\pm 0.2 \mathrm{kPa}$ in the majority of patients. The authors concluded that predicted pressure constitutes a good starting point for CPAP titration, allowing the optimum pressure to be achieved with only a few incremental changes [6].

\section{AFFILIATIONS}

*Dept of Respiratory Diseases and Sleep Medicine Centre, Ghent University Hospital, Ghent, ${ }^{f}$ Service de Pneumologie, Cliniques Universitaires Saint-Luc, Université Catholique de Louvain, Brussels, Belgium.

\#Dept of Respiratory Diseases, San Pedro de Alcántara Hospital, Cáceres,

+Unitat de Biofisica i Bioenginyeria, Facultat de Medicina, Universitat de Barcelona-IDIBAPS, Barcelona,

Spain.

'Service de Pneumologie, CHU de Poitiers, Poitiers, France, and

${ }^{\S}$ Consiglio Nazionale delle Ricerche, Istituto di Biomedicina e Immunologia Molecolare, Palermo, Italy.

CORRESPONDENCE

D. Pevernagie

Dept of Respiratory Diseases and

Sleep Medicine Centre

Ghent University Hospital

De Pintelaan 185

9000 Ghent

Belgium

Fax: 3292402341

E-mail: Dirk.Pevernagie@UGent.be

STATEMENT OF INTEREST

The present article was presented as part of the international symposium "Respiratory somnology: a clinical update", sponsored by GlaxoSmithKline, Belgium 0 . Marrone has received reimbursements from Weinmann for attending symposia. 
Auto-adjustable CPAP (A-CPAP) devices are designed to detect the presence (or absence) of respiratory disturbances and to adjust the pressure in response to the appearance (or disappearance) of certain respiratory events [7]. The introduction of A-CPAP devices on the market has revolutionised the prescription of nasal CPAP. Many practitioners have abandoned the classical CPAP titration approach and prescribe A-CPAP using a pressure range between reasonable lower and upper pressure limits. However, considerable doubt exists as to whether these devices are safe and efficacious [8].

The aim of the present article is to review recent evidence with respect to the principles and practice of nasal CPAP therapy for obstructive sleep-disordered breathing (SDB). Issues are being addressed regarding the assessment of therapeutic pressure levels, and the necessary end-points to take into account when performing titration procedures. Benefits and limitations of A-CPAP treatment are reviewed, including aspects of evidence-based medicine, the place of benchmark testing and differences in performance of A-CPAP devices that use different operational algorithms.

\section{MANUAL, AUTO-ADJUSTABLE OR PREDICTED FORMULA OPTIONS FOR CPAP TITRATION}

Nasal CPAP is the most effective treatment in patients with OSAHS [3, 9, 10]. Pressure titration during an attended laboratory polysomnography is often performed to identify an effective pressure level to control apnoeas, hypopnoeas, snoring and respiratory arousals. While this procedure is still standard practice in many laboratories for the assessment of individual CPAP treatment needs, polysomnographic titration is expensive and time-consuming. Recent studies have evaluated whether the initiation of CPAP treatment can be simplified, using either A-CPAP devices or prediction formulae to estimate effective F-CPAP levels. If such evidence could be provided, this would open up a perspective for considerable savings in terms of financial costs and reductions in the waiting lists for polysomnography.

Titration with A-CPAP devices has been recommended as an alternative option to overcome these difficulties [11]. Until recently, only A-CPAP titration using polysomnographic recording in a conventional laboratory setting was recommended [12]. This guideline was based on the observation that only few studies had been carried out in CPAP-naïve patients in unattended conditions, and the results were contradictory [13-16].

The prediction of CPAP pressure by a formula that includes the apnoea/hypopnoea index (AHI) and anthropometric parameters, such as body mass index and neck circumference, has been proposed to simplify conventional CPAP titration. Favourable results using this technique have been published $[6,17]$. Yet another study, using a randomised, crossover, controlled design and including 18 CPAP-naïve patients, showed that efficacy, clinical improvement and treatment adherence outcomes were similar in patients initiated on FCPAP using a prediction formula titration and self-adjustment at home, as compared with standard titration [18].

Recently, a large multicentre study was conducted in Spain to assess whether CPAP titration performed with an unattended domiciliary A-CPAP device, or with a prediction formula, was as effective as conventional CPAP titration performed using full polysomnography [19]. Patients with OSAHS (AHI >30) requiring $\mathrm{CPAP}$ treatment were randomised into the following three groups: 1) standard CPAP titration in the sleep laboratory, 2) A-CPAP titration performing only a single night study at home, and 3) prediction formula CPAP set-up with domiciliary adjustment according to the observation of residual snoring and apnoeas by the partner. The sample size was 360 patients (120 per group), and the follow-up period was 12 weeks.

The Epworth Sleepiness Scale and the polysomnographic variables (AHI, arousal index, percentages of sleep periods and oxygen saturation) showed a similar statistical improvement after CPAP treatment in the three groups. The residual AHI under CPAP treatment was slightly higher in the prediction formula group. There were no statistical differences in the CPAP compliance between groups. With respect to secondary effects, there were no important differences between the standard and the other groups. Nevertheless, there was a tendency for the A-CPAP group to present more side-effects.

The results of this study [19] demonstrate that the routine standard polysomnographic CPAP titration is not mandatory in most patients with OSAHS. Although the prediction formula group had a higher residual AHI, this was not translated in differences regarding clinical scores. In conclusion, A-CPAP or prediction formula titration with domiciliary adjustment can be proposed as reliable alternatives for the conventional procedure. Omitting this procedure could lead to important economical savings and reduction in waiting lists to perform sleep studies, without negatively affecting treatment outcomes.

\section{END-POINTS FOR CPAP TITRATION}

Application of an adequate pressure to the upper airways is required to obtain complete control of SDB. Adequate CPAP represents one of the main factors determining optimal CPAP compliance [10]. However, even the consensus statement proposed in 1994 by the medical section of the American Thoracic Society (ATS) [3] leaves many questions unanswered. In the article by the ATS [3], the following is stipulated: "Titration of CPAP includes recordings of sleep, respiration and oxygenation. The nasal pressure should be raised to a level that eliminates apnoeas, hypopnoeas, desaturation and sleep fragmentation. Various body positions and NREM [nonrapid eye movement] and REM sleep need to be recorded in order to determine the optimal pressure. If oxyhaemoglobin desaturation persists, supplemental oxygen or ventilatory assistance can be administered through a nose mask."

During manual CPAP titration either rapid or slow pressure increments are applied, while observing the patient with respect to subsequent physiological effects on sleep and cardiopulmonary parameters, until the efficient pressure ( $P$ eff) has been reached. This Peff is usually associated with the regression of snoring and electroencephalo-graphic arousals, while normal sleep is restored [20].

It has been demonstrated that CPAP effectiveness is associated with dramatic improvements in morbidity and mortality in patients with OSAHS [21]. However, in the absence of CPAPrelated side-effects, such as nasal obstruction and poor CPAP 
compliance, the persistence of excessive daytime sleepiness has been confirmed in some patients, even after long-term use [22]. This has led to the hypothesis that, at CPAP levels that control obstructive apnoeas and hypopnoeas, sleep disturbance could persist as a consequence of residual elevated upper airway resistance, inducing increased respiratory efforts and associated effort-related arousals. Some authors have argued that the determination of the efficient pressure should be based on the normalisation of respiratory effort. This goal is achieved by increasing the CPAP to a level that eliminates the progressive inspiratory oesophageal pressure swings that occur during obstructive events [20]. CONDOS et al. [23] demonstrated that elevated upper airway resistance can be inferred from the contour of the inspiratory flow tracing. Episodes of inspiratory flow limitation, evidenced by a plateau at the inspiratory part of the respiratory flow tracing, disappear when CPAP pressure level is further increased $[20,23]$.

According to these results, MEURICE et al. [24] investigated whether correction of inspiratory flow limitation during nasal CPAP therapy could have clinical benefits. The evolution of nocturnal polysomnographic parameters and objective daytime vigilance (maintenance of wakefulness tests; MWT) was compared in two groups of OSAHS patients. In the first group, Peff was determined on the regression of apnoea, hypopnoea and snoring in one group, while the Peff level was set to suppress flow limitation in the other group. The evaluation of CPAP therapy was performed after a 3-week period of home treatment at a Peff level that depended on the group they were randomly allocated to. A similar improvement was found in both groups in terms of apnoea/hypopnoea regression and nocturnal oxygenation saturation improvement, as well as normalisation of mean MWT values. However, the two groups were different in terms of variance of MWT values, with a significantly greater scattering of final results in the group of patients treated with a conventionally determined Peff level, when flow limitation was not suppressed by nasal CPAP, corresponding to a less homogeneous improvement of objective daytime performances in this case. All the patients treated with a nasal CPAP level that also controlled flow limitation had total normalisation of individual MWT values, while the MWT values of $30 \%$ of the patients in the other group remained at a low value. Furthermore, a positive correlation was found between final MWT values and the duration of CPAP use for all patients. It was speculated that the regression of flow limitation accounted for the better compliance with CPAP therapy.

These results provide evidence for the necessity to take into account the detection and correction of flow limitation during CPAP titration. While the assessment of flow limitation may not be a routine procedure, as it requires the use of a pneumotachograph, this information is most helpful in the differential diagnosis of persistent daytime somnolence during CPAP therapy using a classical determination of Peff, in the absence of any other aetiology of daytime somnolence. Furthermore, these results would favour the use of A-CPAP devices that detect the presence of inspiratory flow limitation in patients with poor CPAP compliance or in whom daytime somnolence is insufficiently controlled.

\section{USEFULNESS OF BENCHMARK TESTING}

A-CPAP devices aim at constantly adapting the nasal pressure to eliminate SDB. A-CPAP is designed for two potential applications: either for titrating the F-CPAP level required by the patient or for treating the patient with a nasal pressure continuously adapting to emerging sleep breathing disturbances. Regardless of their final application, A-CPAP devices are based on three complementary but conceptually different software sections devoted to the following: 1) detection of "true" respiratory events (apnoeas, hypopnoeas, episodes with flow limitation, episodes with elevated upper airway impedance, snoring, etc.), 2) identification of "false" events or artefacts (sighs, swallowing, cough, speaking, arousals, mouth breathing, air leaks, etc.) and 3) modification of nasal pressure in accordance with event and artefact detection. At present there are no standardised definitions of events/artefacts and there is no agreement on the best procedure to modify pressure as a function of breathing disturbances. Therefore, the manufacturers of A-CPAP devices implement their own proprietary algorithms, the details of which are usually kept undisclosed for commercial reasons. Accordingly, the available A-CPAP devices appear as "black boxes" able to modify nasal pressure according to rules not clearly explained to the sleep clinician [8]. A conventional and necessary approach is to test the effectiveness of A-CPAP devices by means of clinical studies. However, there is considerable inter- and intra-patient variability in addition to the complex nature of sleep breathing disturbances. Consequently, clinical studies are useful to test the end-point effectiveness of a given A-CPAP device, but not to ascertain whether it correctly identifies events and modifies pressure according to the expectations of the sleep clinician. Bench testing is a useful procedure to analyse the performance of A-CPAP devices. The device is connected to a patient simulator that realistically reproduces well-defined sleepdisturbed breathing patterns. A bench test allows assessment of whether a given A-CPAP device adequately detects respiratory events and responds according to a defined strategy to adapt pressure to the breathing pattern. Moreover, bench testing allows the comparison of different A-CPAP devices in terms of response accuracy, by submitting them to exactly the same patterns of disturbed breathing. The patient simulator for A-CPAP bench testing can be implemented following different approaches [25-28]. One of these approaches is to incorporate a collapsible airway segment to mimic the patient's upper airway in such a way that the breathing pattern depends on the nasal pressure applied [28]. With this method, the ability of simulating a wide variety of realistic breathing events under controlled conditions is limited by the physical parameters of the collapsible segment. Another approach is to simulate the patient by implementing a flow- and impedancecontrolled generator [26, 27]. Such a closed-loop procedure allows the easy definition of a simulated upper airway exhibiting both actual events (flow, obstruction and snoring) recorded from patients, and patterns of sleep disturbances with real-time dependence on the pressure applied by the A-CPAP device under test $[26,27]$. The data reported by the different bench tests of commercially available A-CPAP devices show considerable differences in response patterns [25-29].

To date, bench tests have focused on the study of simulated "true" respiratory disturbances. Essentially no information is 
available on how A-CPAP devices perform when it comes to artefact rejection. Artefacts, as described above, may confuse the operational algorithm of the device and cause unnecessary pressure surges [30]. Additional bench tests should therefore be developed that simulate these false events and assess how they are handled by different machines.

A detailed understanding of the behaviour of A-CPAP machines under well-controlled bench test conditions is a first step to evaluate the performance of these devices and to interpret their clinical outcome in patient studies.

\section{DO DIFFERENT A-CPAP DEVICES PERFORM EQUALLY?}

A-CPAP devices are intended to deliver changing pressure levels to adapt to variable pressure needs during sleep in patients with OSAHS $[12,31]$. During wakefulness pressure should be minimal, while during sleep upper airway obstruction should be prevented with the lowest possible pressure. Accordingly, A-CPAP devices should control the associated sleep disruption, ensure a good comfort and compliance to treatment, and, when used in the sleep laboratory, they should be able to provide indications about the optimal therapeutic pressure level to be delivered by F-CPAP machines. Different A-CPAP machines may have a different efficacy on each of these aspects. Thus, performance of a machine can be considered good or bad, depending on which aspects are being considered.

The first A-CPAP prototype was developed in the early 1990s [7]. Since then, a large number of models have been created, which differ greatly from each other (see section on benchmark testing). Therefore, one would anticipate finding large differences in the performance of such machines.

However, the comparison between different A-CPAP devices has proven more difficult than might first be expected. As technology is rapidly evolving, the available studies deal with devices that are often no longer commercialised. Little is being published about newer, and possibly more efficient, machines. As a rule, manufacturers do not disclose the operational algorithms of their A-CPAP devices, which prevents independent researchers from assessing whether event detection and pressure responsiveness is adequate [8]. Therefore, the evaluation of the accuracy of A-CPAP devices relies either upon feeding reproducible events to the devices in an experimental setting, so-called bench testing, or upon the presence of residual disturbed breathing events in ordinarily treated patients.

Studies on patients with OSAHS showed that levels and variability of pressure delivered by the A-CPAP devices can be different [32-36], sometimes to a considerable extent. However, despite differences in pressure output the effects on polysomnographic variables prove to be similar for most ACPAP devices. All of the published reports demonstrate effectiveness in reducing, and usually normalising, obstructive respiratory disorders and sleep fragmentation [11, 33-35, 3747]. Although in some reports significant differences were found, they were small in absolute terms. Besides, sleepiness, as evaluated by the Epworth sleepiness scale, was reduced by all A-CPAP devices as much as by F-CPAP [33, 41-44, 48-50]. These findings suggest that differences in delivered pressures are not paralleled by differences in efficacy in correcting patients' SDB. Comparative trials of A-CPAP versus F-CPAP, as well as between different A-CPAP devices with respect to compliance and subjective preference, have provided equivocal results. Some studies report similar effects, while others point out significant differences [33, 36, 40-43, 45-50]. Once again, when differences were found, they were often small and appeared unrelated to delivered pressure levels.

The studies published to date indicate that correction of respiratory disorders and satisfaction of the patients with treatment are quite similar with different A-CPAP devices, despite their differences in mode of operation and delivered pressures. This apparent paradox may, in part, be explained by methodological and design issues, which prevent the results of different studies from being matched to each other. Conversely, one can assume that in patients with OSAHS who require low therapeutic pressure levels (e.g. between $0.4-$ $0.8 \mathrm{kPa})$, the use of A-CPAP devices will offer little or no additional benefit. Indeed, patients with a flat dose-response curve will probably do better with a F-CPAP device than with an A-CPAP device that varies the pressure between 0.4 and, say, $2.0 \mathrm{kPa}$. When an A-CPAP machine delivers supratherapeutic pressures, the AHI will not be further reduced. Excess pressure may be well tolerated by some patients, up to a point where mouth leaks, mask leaks or other undesirable effects occur. Differences between machines could be diluted in the average OSAHS population, while they could be more evident in patient subgroups with peculiar characteristics, not yet identified. Regarding the subjective appreciation of the therapeutic effects and comfort of treatment, patients may not be aware of the beneficial or adverse effects of any given ACPAP device, since they are asleep when using the device. They will have a better recall of these effects when they are awake. Therefore, reports on subjective evaluation of A-CPAP devices may be biased by the experience the patients have before falling asleep or during episodes of wakefulness after sleep onset. Finally, personal appreciation of these devices may be influenced by personality and by the help and motivation provided by the team of healthcare professionals more than by characteristics of the machine itself [51].

\section{A-CPAP TREATMENT AS A SUBJECT OF CLINICAL TRIALS}

Phase I clinical trials, the first of four phases of clinical trials, are designed to establish the effects of new drugs on humans and are conducted on small populations of generally healthy humans (usually 20-80 people) to specifically determine a drug's toxicity, absorption, distribution and metabolism. They should follow a phase of pre-clinical studies where the potential new therapeutic drug is subjected to chemical and animal studies to define its therapeutic interest. In phase II studies, a drug having already successfully passed a phase I study is tested for safety and efficacy in a slightly larger population of individuals (usually 100-300 people) who are affected with the disease or condition for which the drug was developed. Phase III studies are conducted on large populations of affected patients (usually $>1,000$ people). They aim to test the new drug in comparison with the standard treatment currently being used for the disease in question. The results of phase III trials usually provide the information that is included in the package insert and labelling. Phases II and III clinical trials often include a comparison with placebo, provided the 
ethical issues related to the use of a placebo in patients are adequately taken into account. Phase IV studies are conducted after a drug has been approved for human use, to compare the drug with a competitor, explore additional patient populations or to further study adverse effects.

CPAP is not a drug, but a medical device. These devices are also subject to administrative rules before they can be launched on the market. Safety, efficacy, side-effects and potential risks have to be studied and/or demonstrated, and post-marketing surveillance needs to be implemented. Rules are stricter for devices designed to be permanently implanted in the body, but also apply to nonimplantable devices.

In fact, when CPAP was introduced for the treatment of OSAHS in the early 1980s, rules for medical devices were not very strict. CPAP devices were released on the market without going through a rigorous assessment based on randomised clinical trials. Long-term safety issues or infectious risks were not assessed. As a logical consequence, placebo-controlled trials were never organised by the industrial manufacturers. It was only after the publication of a review pointing to the lack of medical evidence for the efficacy and effectiveness of CPAP in patients with OSAHS [52] that the "OSAHS community" mobilised to produce a methodologically sound series of convincing proofs.

In a similar manner to the development of machines delivering F-CPAP, A-CPAP devices began to be produced and marketed in a rather anarchic way, without any serious or formalised process of clinical trials demonstrating their added value. Each manufacturer designed a device based on the monitoring of some physiological signal(s) and using proprietary algorithms. No two machines are comparable in design, signals monitored, speed of pressure adjustment, speed of pressure decrease after normalisation of breathing or any other technical characteristic [26]. Each manufacturer tried to demonstrate the equivalence between the new A-CPAP and the old F-CPAP in terms of normalising sleep and breathing, and the superiority of the new A-CPAP device in needing "less pressure" to obtain the same beneficial effect. Hence, many published reports stress that with A-CPAP devices, the mean nocturnal pressure is lower than with F-CPAPs. They systematically omit to mention that with A-CPAP devices the maximal pressure (and frequently the 95th percentile pressure) is higher than with F-CPAP devices [53]. Moreover, they offer no proof that this difference is relevant for short-term physiological or for longterm clinical outcomes. Placebo-controlled trials of the ACPAP technology are not available. Long-term safety data are scarce, if there are any at all. Finally, the positioning of CPAP technology remains unclear: are these devices intended as long-term home treatment devices? Or rather, are they cheap laboratory or home titration devices?

Until recently, the absence of formalised clinical trials could seem unimportant for a treatment aiming at reducing or normalising excessive daytime sleepiness; however, the landscape has now changed. CPAP not only reduces, almost from the first night of use, excessive daytime sleepiness; it also appears to improve survival in the long-term [21]. This sets a new standard to which the A-CPAP technology should be compared in a rigorous, formal and standardised way.
The long history of CPAP makes the planning and performance of phase I studies unnecessary. Similarly, a large number of patients have been treated worldwide for years, with some data reported on long-term compliance and sideeffects. This "unplanned" database could be considered a postmarketing phase II-III study.

However, nothing of the sort is available for A-CPAP devices. If these devices are to be used as titrating machines [32], for very short periods of time, two problems remain to be solved. First, manufacturers should be able to prove that their devices perform as described or claimed. Recent bench studies cast doubt on this issue and have shown that some devices do not correct some abnormal breathing patterns they are supposed to treat $[26,28,54]$. Secondly, manufacturers should be able to prove that their machines do not react to normal physiological variations in breathing during sleep. Unpublished data seem to show that at least some A-CPAP devices increase pressure when applied to polysomnographically proven strictly normal subjects. Thirdly, inappropriate pressure adjustment may ensue from extraordinary events, such as movement, swallowing, coughing and mask and mouth leaks. The solution to these three problems could require formal studies akin to phase I-II trials, in addition to bench studies.

If A-CPAPs are to be used as treatment devices for long-term home therapy of OSAHS, then much more stringent criteria should prevail. Indeed, each manufacturer of an A-CPAP device should prove, in a long-term (5 yrs would seem a reasonable time scale) randomised controlled phase III trial, that their particular A-CPAP device reduces total and cardiovascular mortality to the same extent or more than a traditional F-CPAP comparator. If this kind of study became mandatory, their cost would be very significant, and should impact the final cost of any A-CPAP device.

Based on the above arguments, the absence of phase I-III clinical trials in the A-CPAP realm no longer seems justified.

\section{CONCLUSIONS}

\section{What do we know about current positive airway pressure systems?}

The application of F-CPAP continues to be the gold standard for treatment of moderate-to-severely affected patients with obstructive SDB. Evidence is available that the pressure should be adjusted to a level where not only apnoeas and hypopnoeas are controlled, but where inspiratory flow limitation is also eliminated. It is now well accepted that the use of empirically set CPAP or automatic CPAP devices provides clinical results that are similar to those obtained with the classical approach of manual CPAP titration. The fact that automatic CPAP devices perform very differently on the bench, but still yield satisfactory results on several clinical outcomes, is still an unexplained paradox. Fundamental to the poor knowledge about the efficacy of A-CPAP is the absence of phase I-III clinical trials and the lack of information provided by the industry on the mode of action of their equipment.

\section{What should we expect from new A-CPAP machines?}

Future A-CPAP machines should perform better by simultaneous measuring and integrating signals from different parameters, e.g. flow limitation, upper airway impedance and 
snoring. The integration of different indicators, e.g. increased upper airway impedance during phases of inspiratory flow limitation, will probably result in a more accurate identification of events. Differentiation between true and false respiratory events seems crucial for the proper operation of these devices. Suitable pressure adjustment algorithms should prevent pressure overshooting or undertreatment at any time. Correct identification and management of artefacts should obviate inappropriate pressure responses from these devices.

Finally, manufacturers should abandon the black box concept of new auto-adjustable continuous positive airway pressure equipment released on the market. Physicians who prescribe auto-adjustable continuous positive airway pressure must know when, why and how pressure is being adapted in individual obstructive sleep apnoea/hypopnoea syndrome patients. This information should be logged in the electronic memory of the devices, and be available for download and subsequent review by the healthcare provider. This information will also prove valuable for future studies assessing the performance of auto-adjustable continuous positive airway pressure devices. In this way, it may be determined if some of these devices may be more appropriate than others for particular purposes. Indeed, the choice of equipment may depend on the treatment setting, e.g. titration versus home treatment, aid for habituation in the start-up period versus chronic steady treatment and application in the general OSAHS population versus particular subgroups of patients.

\section{REFERENCES}

1 Sullivan CE, Issa FG, Berthon-Jones M, Eves L. Reversal of obstructive sleep apnoea by continuous positive airway pressure applied through the nares. Lancet 1981; 1: 862-865.

2 Gold AR, Schwartz AR. The pharyngeal critical pressure. The whys and hows of using nasal continuous positive airway pressure diagnostically. Chest 1996; 110: 1077-1088.

3 American Thoracic Society. Indications and standards for use of nasal continuous positive airway pressure (CPAP) in sleep apnea syndromes. American Thoracic Society. Official statement adopted March 1994. Am J Respir Crit Care Med 1994; 150: 1738-1745.

4 Miljeteig $\mathrm{H}$, Hoffstein V. Determinants of continuous positive airway pressure level for treatment of obstructive sleep apnea. Am Rev Respir Dis 1993; 147: 1526-1530.

5 Stradling JR, Hardinge M, Paxton J, Smith DM. Relative accuracy of algorithm-based prescription of nasal CPAP in OSA. Respir Med 2004; 98: 152-154.

6 Oliver Z, Hoffstein V. Predicting effective continuous positive airway pressure. Chest 2000; 117: 1061-1064.

7 Berthon-Jones M. Feasibility of a self-setting CPAP machine. Sleep 1993; 16: Suppl. 8, S120-S121.

8 Brown LK. Autotitrating CPAP: How shall we judge safety and efficacy of a "black box"? Chest 2006; 130: 312-314.

9 Ballester E, Badia JR, Hernandez L, et al. Evidence of the effectiveness of continuous positive airway pressure in the treatment of sleep apnea/hypopnea syndrome. Am J Respir Crit Care Med 1999; 159: 495-501.

10 Loube DI, Gay PC, Strohl KP, Pack AI, White DP, Collop NA. Indications for positive airway pressure treatment of adult obstructive sleep apnea patients: a consensus statement. Chest 1999; 115: 863-866.

11 Teschler H, Berthon-Jones M, Thompson AB, Henkel A, Henry J, Konietzko N. Automated continuous positive airway pressure titration for obstructive sleep apnea syndrome. Am J Respir Crit Care Med 1996; 154: 734-740.

12 Berry RB, Parish JM, Hartse KM. The use of auto-titrating continuous positive airway pressure for treatment of adult obstructive sleep apnea. Sleep 2002; 25: 148-173.

13 Stradling JR, Barbour C, Pitson DJ, Davies RJ. Automatic nasal continuous positive airway pressure titration in the laboratory: patient outcomes. Thorax 1997; 52: 72-75.

14 Berkani M, Lofaso F, Chouaid C, et al. CPAP titration by an auto-CPAP device based on snoring detection: a clinical trial and economic considerations. Eur Respir J 1998; 12: 759-763.

15 Fletcher EC, Stich J, Yang KL. Unattended home diagnosis and treatment of obstructive sleep apnea without polysomnography. Arch Fam Med 2000; 9: 168-174.

16 Series F. Accuracy of an unattended home CPAP titration in the treatment of obstructive sleep apnea. Am J Respir Crit Care Med 2000; 162: 94-97.

17 Rowley JA, Tarbichi AG, Badr MS. The use of a predicted CPAP equation improves CPAP titration success. Sleep Breath 2005; 9: 26-32.

18 Fitzpatrick MF, Alloway CE, Wakeford TM, MacLean AW, Munt PW, Day AG. Can patients with obstructive sleep apnea titrate their own continuous positive airway pressure? Am J Respir Crit Care Med 2003; 167: 716-722.

19 Masa JF, Jimenez A, Duran J, et al. Alternative methods of titrating continuous positive airway pressure: a large multicenter study. Am J Respir Crit Care Med 2004; 170: 1218-1224.

20 Montserrat JM, Ballester E, Olivi H, et al. Time-course of stepwise CPAP titration. Behavior of respiratory and neurological variables. Am J Respir Crit Care Med 1995; 152: 1854-1859.

21 Marin JM, Carrizo SJ, Vicente E, Agusti AG. Long-term cardiovascular outcomes in men with obstructive sleep apnoea-hypopnoea with or without treatment with continuous positive airway pressure: an observational study. Lancet 2005; 365: 1046-1053.

22 Bedard MA, Montplaisir J, Malo J, Richer F, Rouleau I. Persistent neuropsychological deficits and vigilance impairment in sleep apnea syndrome after treatment with continuous positive airways pressure (CPAP). J Clin Exp Neuropsychol 1993; 15: 330-341.

23 Condos R, Norman RG, Krishnasamy I, Peduzzi N, Goldring RM, Rapoport DM. Flow limitation as a noninvasive assessment of residual upper-airway resistance during continuous positive airway pressure therapy of obstructive sleep apnea. Am J Respir Crit Care Med 1994; 150: 475-480.

24 Meurice JC, Paquereau J, Denjean A, Patte F, Series F. Influence of correction of flow limitation on continuous positive airway pressure efficiency in sleep apnoea/ hypopnoea syndrome. Eur Respir J 1998; 11: 1121-1127.

25 Lofaso F, Lorino AM, Duizabo D, et al. Evaluation of an auto-nCPAP device based on snoring detection. Eur Respir J 1996; 9: 1795-1800. 
26 Farre R, Montserrat JM, Rigau J, Trepat X, Pinto P, Navajas D. Response of automatic continuous positive airway pressure devices to different sleep breathing patterns: a bench study. Am J Respir Crit Care Med 2002; 166: 469-473.

27 Rigau J, Montserrat JM, Wohrle H, et al. Bench model to simulate upper airway obstruction for analyzing automatic continuous positive airway pressure devices. Chest 2006; 130: 350-361.

28 Abdenbi F, Chambille B, Escourrou P. Bench testing of auto-adjusting positive airway pressure devices. Eur Respir J 2004; 24: 649-658.

29 Lofaso F, Desmarais G, Leroux K, et al. Bench evaluation of flow limitation detection by automated continuous positive airway pressure devices. Chest 2006; 130: 343-349.

30 Marrone O, Insalaco G, Bonsignore MR, Romano S, Salvaggio A, Bonsignore G. Sleep structure correlates of continuous positive airway pressure variations during application of an autotitrating continuous positive airway pressure machine in patients with obstructive sleep apnea syndrome. Chest 2002; 121: 759-767.

31 Roux FJ, Hilbert J. Continuous positive airway pressure: new generations. Clin Chest Med 2003; 24: 315-342.

32 Kessler R, Weitzenblum E, Chaouat A, Iamandi C, Alliotte T. Evaluation of unattended automated titration to determine therapeutic continuous positive airway pressure in patients with obstructive sleep apnea. Chest 2003; 123: 704-710.

33 Senn O, Brack T, Matthews F, Russi EW, Bloch KE. Randomized short-term trial of two autoCPAP devices versus fixed continuous positive airway pressure for the treatment of sleep apnea. Am J Respir Crit Care Med 2003; 168: 1506-1511.

34 Stammnitz A, Jerrentrup A, Penzel T, Peter JH, Vogelmeier C, Becker HF. Automatic CPAP titration with different self-setting devices in patients with obstructive sleep apnoea. Eur Respir J 2004; 24: 273-278.

35 Pevernagie DA, Proot PM, Hertegonne KB, Neyens MC, Hoornaert KP, Pauwels RA. Efficacy of flow- vs impedance-guided autoadjustable continuous positive airway pressure: a randomized cross-over trial. Chest 2004; 126: 25-30.

36 Nolan GM, Ryan S, O'Connor TM, McNicholas WT. Comparison of three auto-adjusting positive pressure devices in patients with sleep apnoea. Eur Respir J 2006; 28: 159-164.

37 Scharf MB, Brannen DE, McDannold MD, Berkowitz DV. Computerized adjustable versus fixed NCPAP treatment of obstructive sleep apnea. Sleep 1996; 19: 491-496.

38 Konermann M, Sanner BM, Vyleta M, et al. Use of conventional and self-adjusting nasal continuous positive airway pressure for treatment of severe obstructive sleep apnea syndrome: a comparative study. Chest 1998; 113: 714-718.
39 Boudewyns A, Grillier-Lanoir V, Willemen MJ, De Cock WA, Van de Heyning PH, De Backer WA. Two months follow up of auto-CPAP treatment in patients with obstructive sleep apnoea. Thorax 1999; 54: 147-149.

40 d'Ortho MP, Grillier Lanoir V, Levy P, et al. Constant vs automatic continuous positive airway pressure therapy home evaluation. Chest 2000; 118: 1010-1017.

41 Planes C, D'Ortho MP, Foucher A, et al. Efficacy and cost of home-initiated auto-nCPAP versus conventional nCPAP. Sleep 2003; 26: 156-160.

42 Meurice JC, Marc I, Series F. Efficacy of auto-CPAP in the treatment of obstructive sleep apnea/hypopnea syndrome. Am J Respir Crit Care Med 1996; 153: 794-798.

43 Hussain SF, Love L, Burt H, Fleetham JA. A randomized trial of auto-titrating CPAP and fixed CPAP in the treatment of obstructive sleep apnea-hypopnea. Respir Med 2004; 98: 330-333.

44 Ficker JH, Fuchs FS, Wiest GH, Asshoff G, Schmelzer AH, Hahn EG. An auto-continuous positive airway pressure device controlled exclusively by the forced oscillation technique. Eur Respir J 2000; 16: 914-920.

45 Randerath WJ, Schraeder O, Galetke W, Feldmeyer F, Ruhle KH. Autoadjusting CPAP therapy based on impedance efficacy, compliance and acceptance. Am J Respir Crit Care Med 2001; 163: 652-657.

46 Teschler $\mathrm{H}$, Wessendorf TE, Farhat AA, Konietzko N, Berthon Jones M. Two months auto-adjusting versus conventional nCPAP for obstructive sleep apnoea syndrome. Eur Respir J 2000; 15: 990-995.

47 Massie CA, McArdle N, Hart RW, et al. Comparison between automatic and fixed positive airway pressure therapy in the home. Am J Respir Crit Care Med 2003; 167: 20-23.

48 Hudgel DW, Fung C. A long-term randomized, cross-over comparison of auto- titrating and standard nasal continuous airway pressure. Sleep 2000; 23: 645-648.

49 Marrone O, Resta O, Salvaggio A, Giliberti T, Stefano A, Insalaco G. Preference for fixed or automatic CPAP in patients with obstructive sleep apnea syndrome. Sleep Med 2004; 5: 247-251.

50 Hukins C. Comparative study of autotitrating and fixedpressure CPAP in the home: a randomized, single-blind crossover trial. Sleep 2004; 27: 1512-1517.

51 Berry RB. Improving CPAP compliance - man more than machine. Sleep Med 2000; 1: 175-178.

52 Wright J, Johns R, Watt I, Melville A, Sheldon T. Health effects of obstructive sleep apnoea and the effectiveness of continuous positive airways pressure: a systematic review of the research evidence. BMJ 1997; 314: 851-860.

53 Rodenstein DO. Automatically controlled continuous positive airway pressure. A bright past, a dubious future. Eur Respir J 2000; 15: 985-987.

54 Lofaso F, Desmarais G, Leroux K, et al. Bench evaluation of flow limitation detection by automated continuous positive airway pressure devices. Chest 2006; 130: 343-349. 